\title{
Estrategia Metaheurística para Redes Ópticas sin Conversión de Longitud de Onda con Tráfico Dinámico (WDM)
}

\author{
Arturo B. Rodriguez ${ }^{(1)}$, Fideromo Saavedra ${ }^{(2)}$ y Leonardo J. Ramírez ${ }^{(3)}$ \\ (1) Universidad Santiago de Chile, Depto. de Tecnología Industrial, Grupo de Investigación en Nuevas \\ Tecnologías (GINT), Santiago-Chile (email: arturo.rodriguez@usach.cl) \\ (2) Universidad Santiago de Chile, Departamento de Ingeniería Eléctrica, Grupo de Investigación Fotónica \\ (GIFOT), Santiago-Chile (email: fideromo.saavedra@usach.cl) \\ (3) Univ. Militar de Nueva Granada, División de Desarrollo Tecnol. e Innov. de la Vicerrectoría de Inv., Grupo \\ de Investigación en Telemedicina (TIGUM), Bogotá, Colombia (email: leonardo.ramirez@unimilitar.edu.co)
}

Recibido Dic. 23, 2015; Aceptado Mar. 2, 2016; Versión final Mar. 17, 2016, Publicado Oct. 2016

\begin{abstract}
Resumen
La presente investigación muestra la comparación entre diferentes heurísticas tales como Algoritmos genéticos, Simulated Annealing, Tabú Search, Snake-One y una nueva metaheurística Snake-Two. Anteriormente se obtuvieron bajos niveles de probabilidad de bloqueo con tendencia de aumento del uso de recursos. La estrategia Snake-Two demuestra que el comportamiento de la probabilidad de bloqueo disminuye a un costo bajo de utilización de la red. Estos resultados son comparados con un algoritmo convencional utilizado como referencia que permite visualizar la mejora en cada indicador. Los indicadores utilizados son la probabilidad de bloqueo y la utilización de la red y un nuevo indicador denominado Tasa de Algoritmo Heurístico. Esta estrategia propone utilizar enlaces congestionados hasta saturarlos permitiendo que el tráfico se concentre en algunas zonas y desdensifique otras. Los resultados son prometedores al lograr disminuir la probabilidad de bloqueo pero se mantiene la tendencia de aumento del uso de los recursos de la red.
\end{abstract}

Palabras clave: recocido simulado; algortimos genéticos; modelo activo de contorno; snake one; snake two

\section{Metaheuristic Strategy for Dynamic Traffic in Wavelength Division Multiplexing Optical Networks (WDM)}

\begin{abstract}
This research shows the performance comparison between different heuristics such as genetic algorithms, simulated annealing, Tabu Search, Snake-One and a new metaheuristic called Snake-Two. Earlier were obtained low blocking probability but with a tendency to increase network utilization. The new Snake-Two strategy can prove that the behavior of the blocking probability decreases at low network usage. These results are compared with a conventional algorithm that was used to visualize the improvement of each indicator. The indicators used for comparison, are the blocking probability and the network utilization and a new indicator called Heuristic Algorithm Rate. This strategy proposes using the congested links, allowing increasing the traffic in some areas, and reducing it in other areas. The results are promising to achieve lower blocking probability but the tendency of increasing the use of the network resources continues.
\end{abstract}

Keywords: simulated annealing; genetics algorithm; active contour model; snakes methods 


\section{INTRODUCCIÓN}

El continuo crecimiento de la demanda de servicios para las redes de transporte tales como SDH (Synchronous Digital Hierarchy) y SONET (Synchronous Optical NETwork) han permitido el desarrollo de las redes ópticas que le dan soporte. Estas redes ópticas se desarrollaron hasta alcanzar lo que se denomina hoy redes WDM (Wavelength Division Multiplexing), estas redes logran multiplexar diferentes longitudes de onda por la misma fibra, las diferentes señales ópticas deben tener un ancho de línea lo suficientemente angosto para permitir la mayor cantidad de señales multiplexadas. Dependiendo de la distancia se pueden clasificar en CWDM (Course (Wavelength Division Multiplexing) y DWDM (Dense (Wavelength Division Multiplexing) para distancias cortas y largas respectivamente. Las redes CWDM utilizan menos de 18 longitudes de onda y las DWDM hasta 160 longitudes de onda, si disminuyen las señales multiplexadas el ancho de línea de las señales puede aumentar lo que implicaría utilizar transmisores (láseres o Led) de menor costo, además de poder trabajar en rangos de transmisión de menor longitud de onda (Juhari et al.,2015; Zahilah, 2013).

Al existir varios canales dentro de una misma fibra y múltiples caminos para transitar por la red, el principal problema es determinar la ruta (conjunto de enlaces) y la longitud de onda para cada solicitud entrante, estas rutas y longitudes de ondas son conocidas como LightPath (LP) y están sometidas a restricciones de continuidad o reutilización de longitud de onda para los escenarios sin y con conversión de longitud de onda respectivamente. Este problema es conocido por sus siglas RWA (Routing Wavelength Assignment) o enrutamiento y asignación de longitud de onda. La evolución del tráfico de transporte de datos llevó a las redes actuales a un escenario de tráfico dinámico, el cual es diferente al tráfico estático que lo antecede.

Antes las solicitudes de servicio tenían un elevado Holding Time o tenencia de la conexión que era mucho mayor que el tiempo entre llegadas de solicitudes, por lo que la disponibilidad de la red, permanecía bastante estática y esto permitía utilizar algoritmos convencionales de optimización para la búsqueda de los lightpath, tales como Dijkstra, Floyd Warshal, Ford-Fulkerson entre otros. Pero, el tráfico dinámico hoy el Holding Time es menor al tiempo entre llegada de solicitudes, como consecuencia ocurre que existe una disponibilidad de la red siempre cambiante y dinámica. Este nuevo escenario no permite el uso de algoritmos convencionales dando espacio para el desarrollo de algoritmia heurística y metaheurística (Chiappone et al., 2016; Rodriguez et al., 2015; Pan et al., 2014; Assis, et al., 2010; Zang, et al., 2001). Los indicadores utilizados para evaluar los diferentes algoritmos son la Probabilidad de Bloqueo (PB), la Utilización de la red (UR) y la tasa de Algoritmia Heurística (TAH). En el presente artículo se muestra una metaheurística que mejora los resultados del algoritmo Snake One, obteniendo resultados positivos para el indicador probabilidad de bloqueo.

\section{ALGORITMOS HEURÍSTICOS}

El problema RWA es conocido como SLE (Static Lightpath Establishment) o Establecimiento estático de rutas de luz y DLE (Dynamic Lightpath Establishment) o Establecimiento dinámico de rutas de luz, para tráfico estático y dinámico según corresponda. Para responder al problema DLE se han utilizado estrategias como: (a) Solución con división del problema en dos partes, el primero encuentra la ruta y el segundo le asigna longitud de onda; y (b) Solución integral, donde se encuentra la ruta y la longitud de onda en el mismo proceso algorítmico. En la Figura 1, se puede observar la esquematización de las diferentes estrategias tales como la dividida y la integral, así como los algoritmos heurísticos utilizados en la solución del RWA.

Se define $N$ como el número de nodos activos en la red óptica, $n_{M C X}$ es el número de conexiones máximo en cada enlace, $n_{C X}$ es el número de conexiones solicitadas, $n_{W}$ es el número de Longitudes de Onda en cada enlace (supuesto iguales en todos los enlaces de la red) y $t_{C X}$ es el tiempo de conexión solicitado (Holding Time. Los nodos reciben un modelo de solicitud (Demanda, $D$ ) de servicios tal como se muestra en la ecuación 1.

$D=\left(\right.$ Origen, Destino, $\left.n_{C X}, t_{C X}\right)$

En la presente investigación se han ensayado algoritmos heurísticos partiendo de un universo de soluciones aleatorio (población inicial):

La población inicial para todos los casos es una Matriz 3D que es llenada aleatoriamente (Ver ecuación 1)

$P_{0}=\left\{\begin{array}{l}p_{i j k} / p_{i j k}=a \quad \forall i \in\left[0, n_{P}-1\right] \wedge j \in\left[0, N+n_{W}+4\right] \wedge k \in[0, N-1] \wedge a \leq[0, N-1] \\ \wedge p_{i 0 k}=\text { Origen } \wedge p_{i(N-1) k}=\text { Destino }\end{array}\right\}$ 


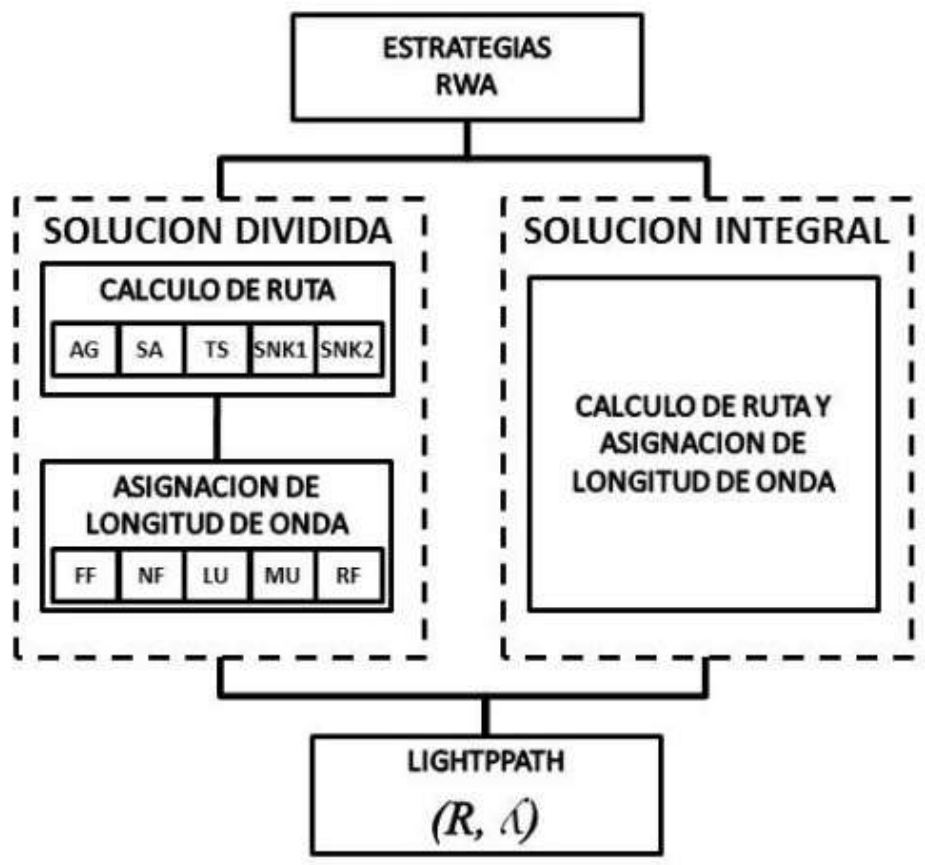

Fig. 1: Solución integral y dividida

En esta ecuación: ( $i, j, k$ es la ubicación del elemento en la matriz poblacional; a es el valor aleatorio que representa a cada nodo de la topología; $n_{P}$ e: es el número de filas de la matriz poblacional; $N$ es el número de nodos de la Red; y $n_{w}$ es el número de longitudes de onda.

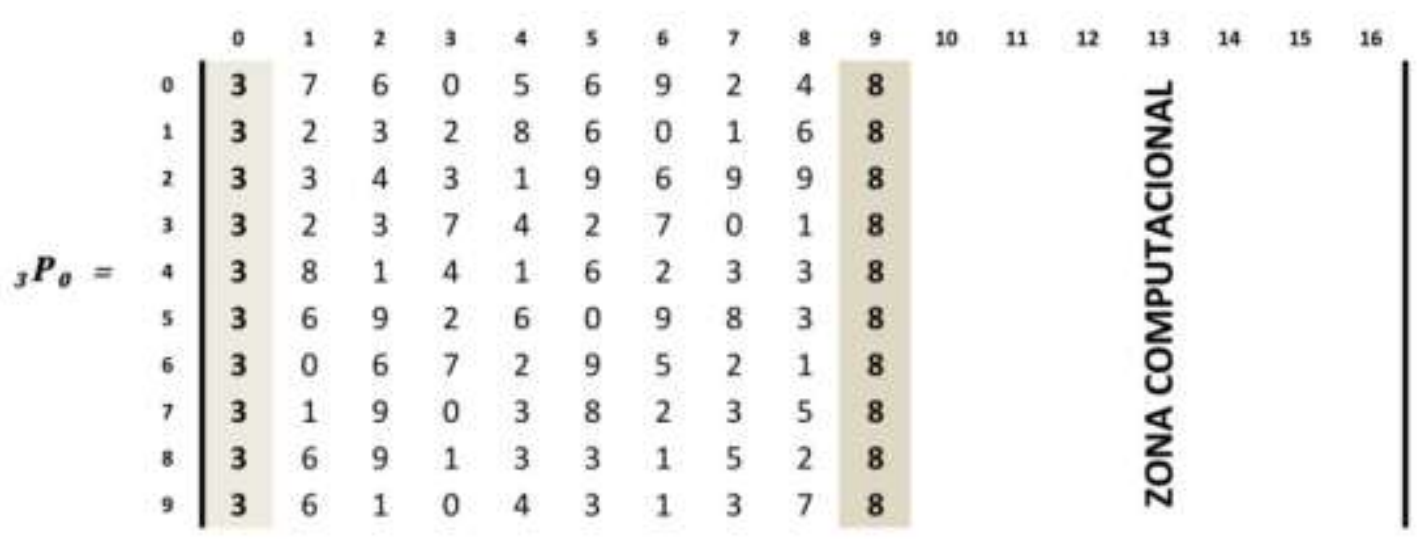

Fig. 2: Matriz poblacional Inicial para la longitud de Onda 0, de una topología de 10 Nodos con 3 Longitudes de Onda, para una solicitud del nodo 3 al nodo 8

En la Figura 2, se muestra un ejemplo de la matriz poblacional inicial para una topología de 10 nodos $(N=10)$ (Ver topología ejemplo en Figura 3) y 10 filas $\left(n_{P}=10\right)$ y 3 longitudes de onda $\left.\left(n_{W}=3\right) \lambda_{0}=0, \lambda_{1}=1, \lambda_{2}=2\right)$. La matriz poblacional inicial mostrada corresponde la matriz del nodo 3 , cada longitud de onda tiene una matriz conformando de esta forma una matriz 3D. El llenado de esta matriz es aleatorio los nodos son etiquetados de 0 a 9 , y se adiciona una columna por cada longitud de onda y 4 columnas para cálculos computacionales auxiliares de los algoritmos.

Los algoritmos genéticos $(A G)$, tienen la característica principal de utilizar cada fila de la matriz poblacional como cromosoma y desarrollar una reproducción basada en el intercambio de genes entre filas, lo que genera nuevos cromosomas resultado de la combinación, estos son utilizados para el cálculo del costo en la matriz guardándose en la zona computacional de la matriz; luego la matriz se ordena de menor a mayor y los cromosomas (filas) empiezan a tener genes (nodos) cada vez más adyacentes en la red, de esta forma el costo comienza disminuir hasta que cae por debajo de un umbral que determina la existencia de la ruta. 


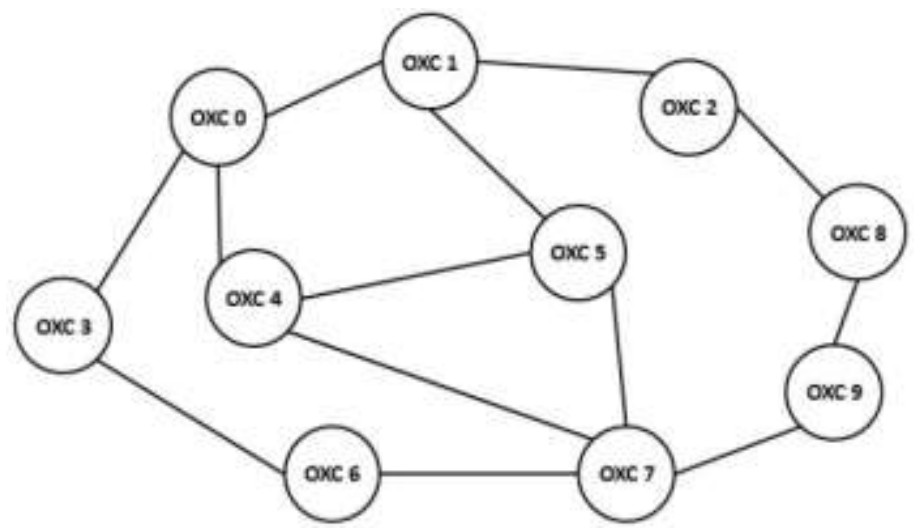

Fig. 3: Red de 10 Nodos y 13 enlaces con 3 longitudes de onda

En el proceso, se eliminan las últimas filas (mortandad) y se rellenan aleatoriamente (natalidad constante), de esta forma se garantizan nuevos genes que pueden ayudar a la convergencia (Rodriguez et al., 2014; Kumar, et al., 2013; Rubio-Largo et al., 2013; Baparanda et al., 2010). El algortimo Simulated Annealing (SA) (Templado Simulado), tiene la característica principal de simular el enfriamiento del acero, utilizando un proceso cíclico y de velocidad regulada, donde la matriz poblacional es dividida en capas desde afuera hacia adentro. Estas capas rotan al mismo paso y horizontalmente se miden los costos de las rutas, guardándose los resultados en la zona computacional, luego se ordena y se sigue el proceso hasta que algunas de las rutas sea inferior al umbral; esto emula el acomodo de los átomos del proceso martensítico para obtener el acero (enfriamiento lento) (Bettemir, et al., 2015; Rodriguez et al., 2011). El algoritmo búsqueda Tabú (TS), tiene la característica principal del intercambio de las columnas de la matriz poblacional donde los cambios generan disminuciones de los costos; si los intercambios producen un decremento del costo de la ruta ese intercambio se premia y si el intercambio produce un aumento en el costo de las rutas se castiga por una cantidad específica de iteraciones que disminuye hasta que vuelve a estar operativo el intercambio de esas columnas. Luego, se ordenan las filas de la matriz poblacional hasta que se logren rutas por debajo del umbral pre-establecido; por lo general este umbral es el costo máximo de la ruta (Rodriguez et al., 2014; Chen et al., 2011). El algortimo Snake One (SNK1), tiene como característica principal la no utilización de la matriz poblacional, disminuyendo la complejidad temporal. Todo el proceso algorítmico se lleva a cabo en la matriz de costos, utiliza desplazamiento horizontal y vertical en la matriz de costos de manera secuencial hasta encontrar el destino, encontrando una ruta funcional no necesariamente óptima (Rodríguez et al., 2015).

Por otro lado, se revisaron diferentes heurísticas con innovadoras propuestas que resuelven el problema RWA como: Ant System (Dong et al., 2015; Chen et al., 2011), Bee Colony (Rubio-Largo et al., 2011), Mutiobjetivo gravitacional (Rubio-Largo et al., 2011), programación paralela (Pandya et al., 2014; ; RubioLargo et al., 2012; Rubio-Largo et al., 2011) y algoritmo luciérnaga (Xin-She et al., 2015); que logran resultados competitivos para el caso SLE (Static Lightpath Establishment) pero que son ineficaces para el caso DLE (Dynamic Lightpath Establishment) .

\section{METAHEURÍSTICA SNAKE TWO}

La estrategia (metaheurística) Snake Two (SNK2) se basa en concentrar el tráfico en algún sector de la red llevando los enlaces a su estado saturado (estado de máxima capacidad) con la finalidad de descongestionar aquellos enlaces que son poco utilizados por el algoritmo. En la Figura 4, se observa la utilización de Snake One para cada extremo del enlace congestionado elegido de la matriz ordenada MEC (Ver ecuación 2).

$M E C=\left\{\begin{array}{l}m_{i j k} / m_{i j k}=C l_{(i, j)} \text { para } k \text { - ésima longitud } \\ \text { de onda, } \forall i \in[0, N(N-1)] \wedge j=3 \wedge k \in\left[0, n_{w}-1\right]\end{array}\right\}$

En la ecuación 2: $(i, j, k)$ es la ubicación del elemento en la Matriz Enlaces Congestionados y $C l_{(i, j)}$ es el costo instantáneo del enlace $(i, j)$, El costo instantáneo es el número de canales disponibles para servicio en cada instante, el mismo que depende de la cantidad de canales que entrega el enlace cuando entra en servicio.

Para lograr esta concentración se debe monitorizar el tráfico de cada enlace y mantener la MEC (Matriz de Enlaces Congestionados) ordenada de tal forma que siempre se tenga en la parte superior un enlace con máximo costo. Por otro lado, el algoritmo deberá buscar el lightpath con la condición de pasar por un enlace de máximo costo (primer enlace en la MEC ordenada), si el número de conexiones solicitadas es superior al 
enlace elegido, entonces deberá pasar al siguiente enlace congestionado. Para lograr que el lightpath pase por el enlace congestionado, se deberá aplicar Snake One (SNK1) del origen a un extremo enlace elegido y luego aplicar Snake One del otro extremo al destino (ver Figura 5).

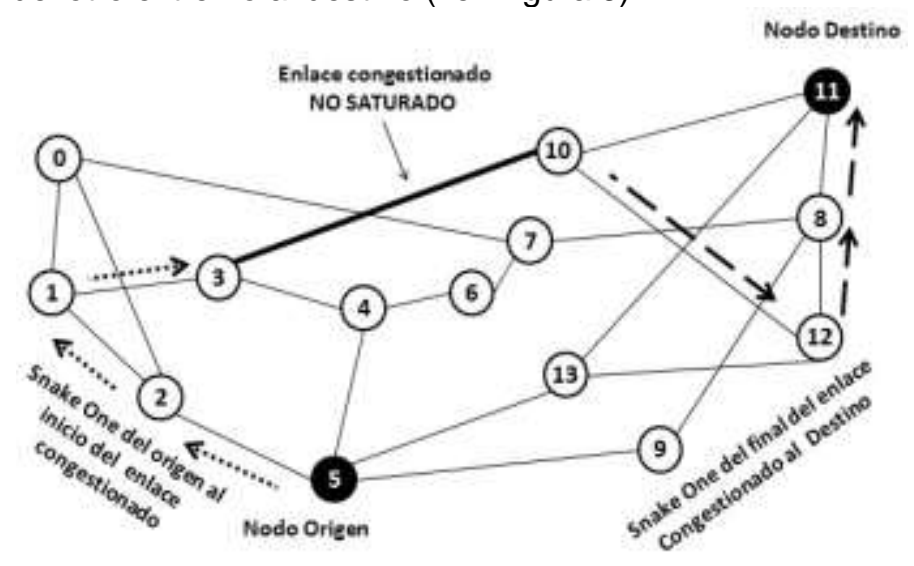

Fig. 4: Meta heurística Snake Two

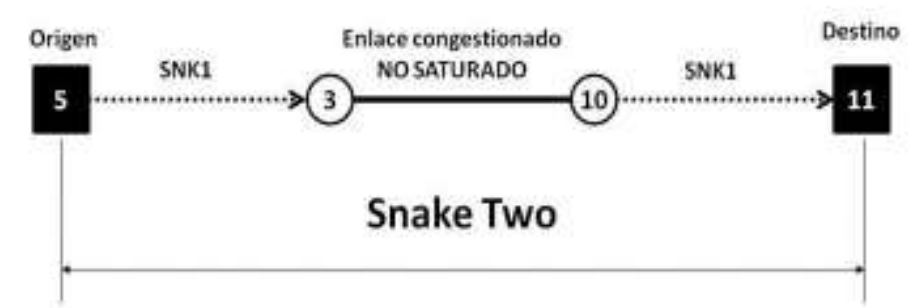

Fig. 5: Esquema Meta heurística Snake Two (SNK2)

La Figura 6, muestra el diagrama de flujo de la metaheurística Snake Two, donde se observa que se inicia con la definición de las matrices $\lambda$ (Control de Lambdas), T (Control de tiempos de conexión), C (de costos), S1 (Matriz de computo de Snake One), y MEC (Matriz de Enlaces congestionados).

Esta última matriz monitoriza el tránsito en los enlaces y la mantiene ordenada con la finalidad de obtener siempre el enlace de mayor congestión, de esta forma siempre se dispondrá de un enlace con la cantidad de conexiones necesarias para satisfacer la solicitud de servicio y además esta estrategia concentra el tráfico en ciertos sectores mientras deja con bajo tráfico otras zonas que servirán para satisfacer la demanda futura. El diagrama de flujo describe la búsqueda de rutas del origen de la solicitud a un extremo del enlace de la MEC seleccionado y del otro nodo de este enlace hacia el destino de la solicitud, con estos tres elementos se compone el LP que satisface la solicitud, en caso que no se logre la composición la solicitud es bloqueada.

Esto se debe a que se obtenían muy altas probabilidades de bloqueo y altos niveles de utilización de la red, por lo extenso de las rutas que obtenía, esta es la razón por la que se limitó a la mitad, solo con la finalidad de que se generen rutas cortas. Si no existen rutas se procede a buscarla en la siguiente longitud de onda, si no hay longitud de onda la solicitud es bloqueada. Al encontrar un lightpath a un extremo se procede a buscar la ruta del otro extremo en la misma longitud de onda (Restricción de continuidad de longitud de onda), si se encuentra ruta con las condiciones dadas entonces se obtiene el Lighpath Solucion, de lo contrario la solicitud es bloqueada.

\section{ESCENARIO DE SIMULACIÓN}

Todos los algoritmos empiezan con una población aleatoria a excepción de Snake One, la misma que utiliza la matriz de costos y genera su propia matriz de desplazamiento (Snake). Para efectos de la simulación se utilizó la red óptica NSFNET (Network Science Foundation NETwork, ver Figura 4) con 14 nodos, 21 enlaces y 8 longitudes de onda (ver Figura 7). La demanda de servicios se distribuye uniformemente para todos los nodos, la demanda de solicitud de servicio es de características poisson, el límite máximo de saltos es la cantidad de nodos por la condición del algoritmo Snake One y se niega el reintento de solicitud cuando esta es bloqueada, condiciones similares para los algoritmos que se estudian en este artículo y que se pueden observar en (Rodríguez et al. 2015; Pan et al., 2014; Zang et al., 2010). 


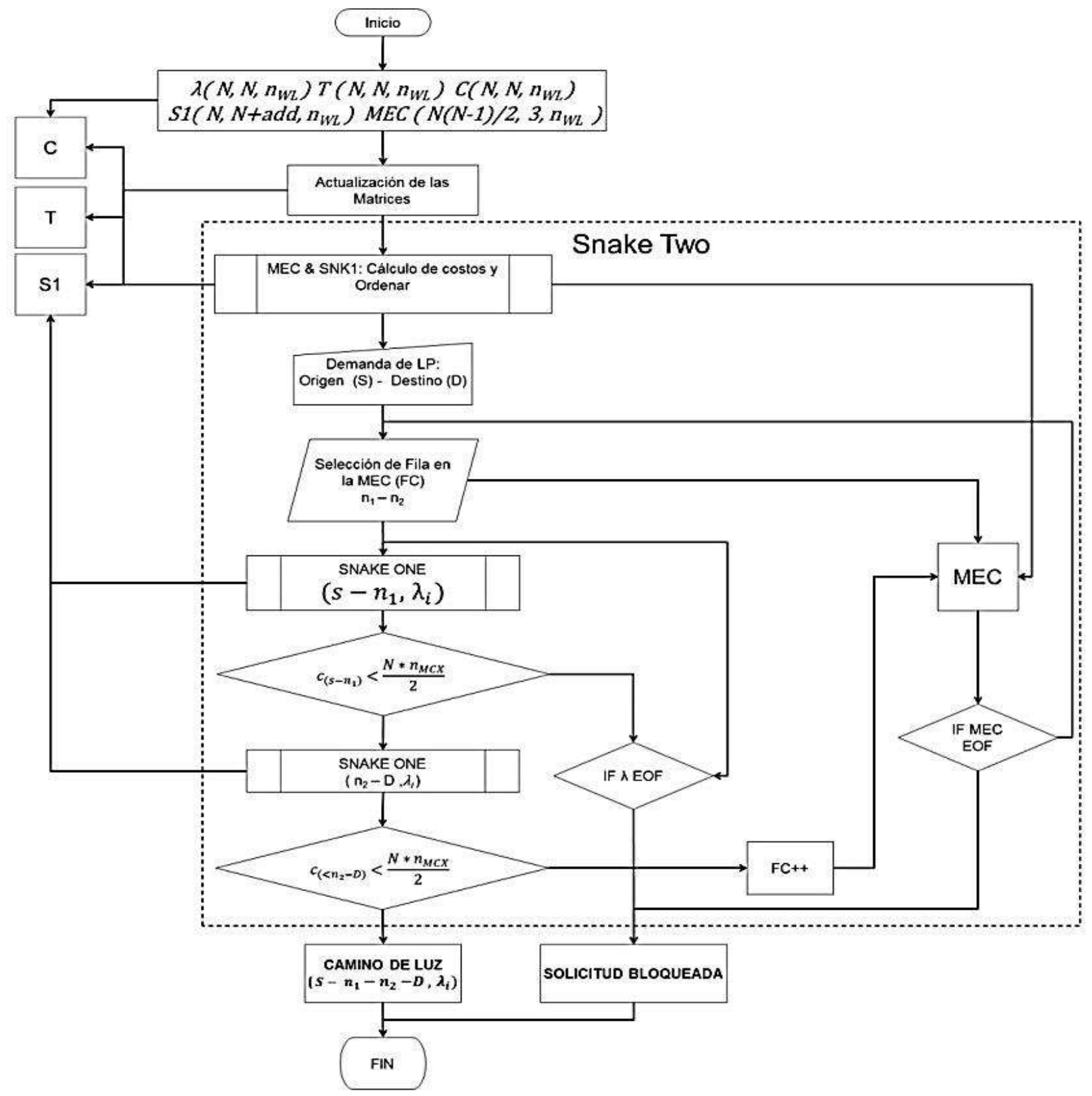

Fig. 6: Diagrama de Flujo de Snake Two

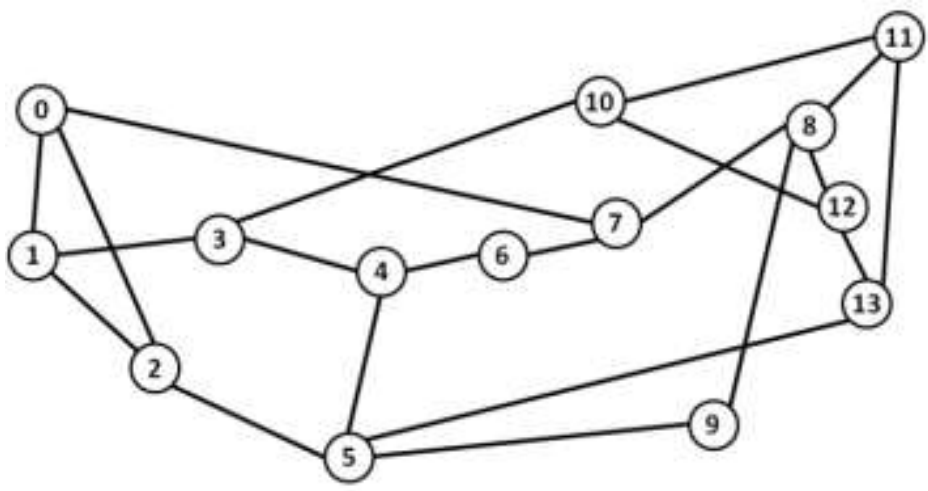

Fig. 7: Escenario de simulación NSFNET (National Science Foundation NETwork).

La simulación se realizó variando las condiciones de tráfico dinámico con cargas hasta los 180 Erlangs cada 10 Erlangs, y se realizaron un total de $10^{8}$ solicitudes de servicio con la finalidad que el tráfico cause stress en la red, cabe indicar que el algoritmo de referencia solo realizó la experiencia hasta los 160 Erlangs. 


\section{ANALISIS DE LOS RESULTADOS}

Para observar el aporte de la metaheurística Snake Two (SNK2) se analizaron comparativamente algoritmos heurísticos, como: Algoritmos genéticos (AG), Simulated Aneealing (SA), Tabú Search (TS) y Snake One (SNK1) y un algoritmo convencional de referencia (RF) (Zang, $\mathrm{H}$ et al., 2001) Para la comparación se calculó los valores medios de cada simulación tanto de la probabilidad de bloqueo como de la utilización de la red. En la tabla 1, se observa una disminución de la probabilidad de bloqueo para la metaheurística Snake Two a costo de elevar la utilización de la red. Así mismo al utilizar el algoritmo de más alta probabilidad de bloqueo (SA) como elemento comparativo porcentual para observar la gradiente negativa (cuanto disminuye) de este indicador, utilizando |PBx-PBSA|PBSA, se hace visible que la estrategia SNK2 (Snake Two) tiene 37\% menos de probabilidad de bloqueo que el de mayor indicador, siendo el que más disminuye inclusive inferior al de referencia.

Tabla 1: Comparación de valores medios (\%)

\begin{tabular}{|l|c|c|c|c|c|c|}
\hline & AG & SA & TS & SNK1 & SNK2 & RF \\
\hline Probabilidad de bloqueo (PB) & 0,42 & 0,45 & 0,36 & 0,32 & 0,28 & 0,32 \\
\hline Utilizacion de la red (UR \%) & 42,17 & 45,3 & 55,17 & 68,2 & 72,22 & 32,17 \\
\hline Gradiente PB (\%) & -7 & 0 & -20 & -29 & -37 & -28 \\
\hline
\end{tabular}

Este comportamiento es positivo porque la demanda futura se ve beneficiada al aumentar su probabilidad de atención. Es necesario visibilizar a cambio de cuanta utilización de la red, la probabilidad de bloqueo disminuye y para ello se elaboró un nuevo indicador denominado Tasa de Algoritmo Heurístico (TAH, ver ecuación 2) que relaciona la probabilidad de bloqueo y la utilización de la red promedio.

$T A H=10^{6} \frac{\overline{P B}}{\overline{U R}}$

EI indicador TAH permite visibilizar cuanto aumentaría en promedio la probabilidad de bloqueo cuando se produce un aumento de la utilización de la red. Podemos observar en la Figura 8, que SNK2 (Snake Two) tiene el menor valor de $\mathrm{TAH}$, lo que indica que en términos de recursos de red, que es el que puede tener mejor condición para enfrentar a la demanda futura, debido a que si aumenta la probabilidad de bloqueo a la red le costará una menor cantidad de recursos. Por otro lado, también se puede observar que las heurísticas AG y SA mantienen un similar comportamiento, lo que indica que sería indiferente utilizar uno u otro para escenarios dinámicos. Además, se observa que el algoritmo de referencia tiene una menor condición frente a la demanda futura, aun siendo un fuerte algoritmo en utilización de la red.

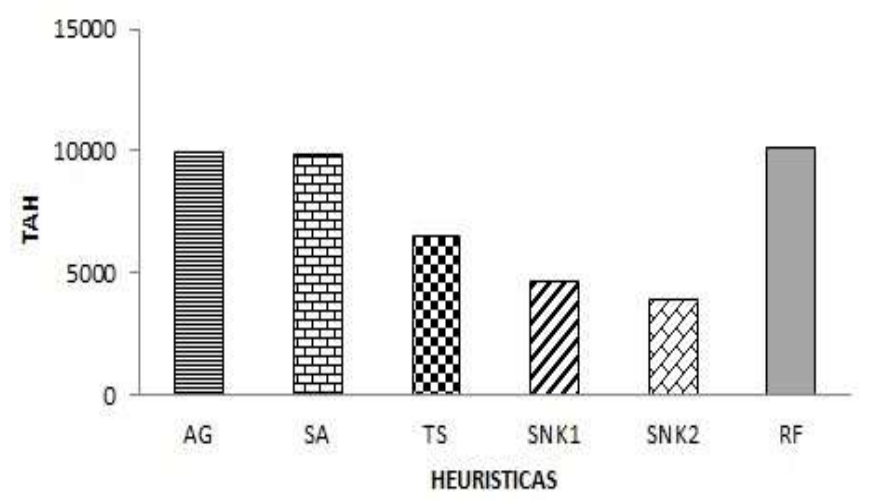

Fig. 8: Comparación de la TAH media para cada algoritmo heurístico.

Observando la Figura 9, vemos que el SNK2 tiene baja disminución de la probabilidad de bloqueo media, lo que indica que la estrategia de concentrar el tráfico saturando los enlaces funciona, sin embargo no logra hacerlo de manera significativa respecto de SNK1 pero si de AG, SA y TS. Por otro lado, considerando que la carga se varió hasta los 180 Erlangs esa disminución tiene relativa importancia para las redes de alto tráfico.

En la Figura 10 podemos observar, la utilización de la red y el aumento del uso de los recursos de SNK2 a pesar de concentrar el uso de los recursos en un grupo de enlaces. Sin embargo, los cambios de la probabilidad de bloqueo en condiciones de stress resultan relevantes toda vez que el aumento del uso de los recursos es bastante bajo. Se debe resaltar el bajo consumo de recursos de los algoritmos AG y SA frente a los algoritmos SNK1 y SNK2; esto se debe a que las rutas son más cortas (menos saltos). 


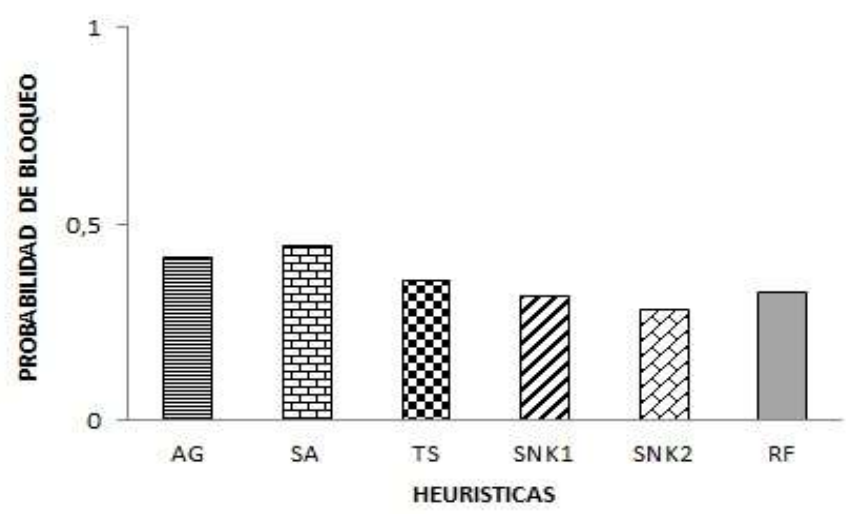

Fig. 9: Comparación de la probabilidad de bloqueo para cada algoritmo heurístico.

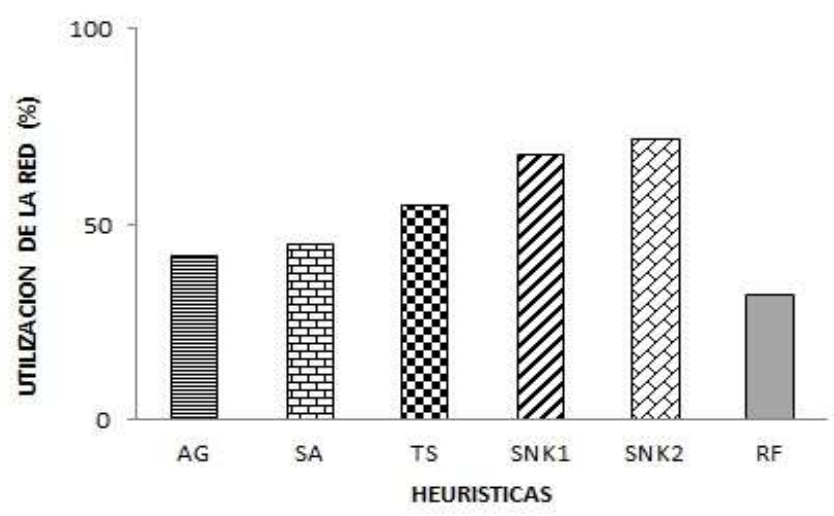

Fig. 10: Comparación de la Utilización de la Red para cada algoritmo heurístico.

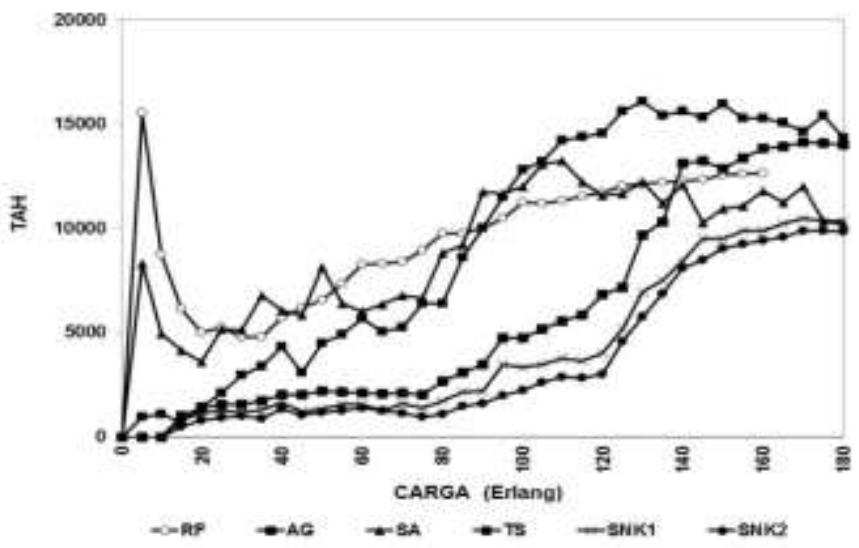

Fig. 11: Comparación de la TAH por carga en Erlangs para cada algoritmo heurístico.

En la Figura 11, se observa el comportamiento de la Tasa de Algoritmo Heurístico para cada carga utilizada en la simulación, es visible que SNK1 y SNK2 se destacan del resto de heurísticas y ofrecen mayor robustez ante cargas muy altas de tráfico. Siendo cierto que AG y SA consumen pocos recursos de la red lo hacen a costa de una alta probabilidad de bloqueo relativa y el TAH lo muestra al aparecer por encima de todas las heurísticas. Además es observable que todas las heurísticas tienden a un estado bastante estable cuando se alcanza un tráfico de 140 Erlangs, esto corresponde al punto de inflexión de la probabilidad de bloqueo observable en la Figura 9.

En la figura 12, se puede observar el comportamiento de la probabilidad de bloqueo del algoritmo SNK2 que logra una mejora poco significativa, pero desde el punto de vista estratégico resulta importante debido a que demuestra que la estrategia de enlace saturado logra modificar el comportamiento de la probabilidad de Bloqueo. Esto motiva a ensayar nuevas metaheurísticas que mejoren estos resultados y permitan un uso menor de la utilización de la red. Hay que resaltar que cuando las heurísticas alcanzan los 140 Erlangs (Stress) son AG y SA. Esto implica que son muy robustoz en escenarios de stress logrando las más bajas 
probabilidades de bloqueo, es decir mayor atención. Pero si observamos el escenario por debajo de los 140 Erlangs SNK2 logra comportarse con muy baja probabilidad de bloqueo frente a las otras heurísticas e inclusive mejor que el algoritmo de referencia.

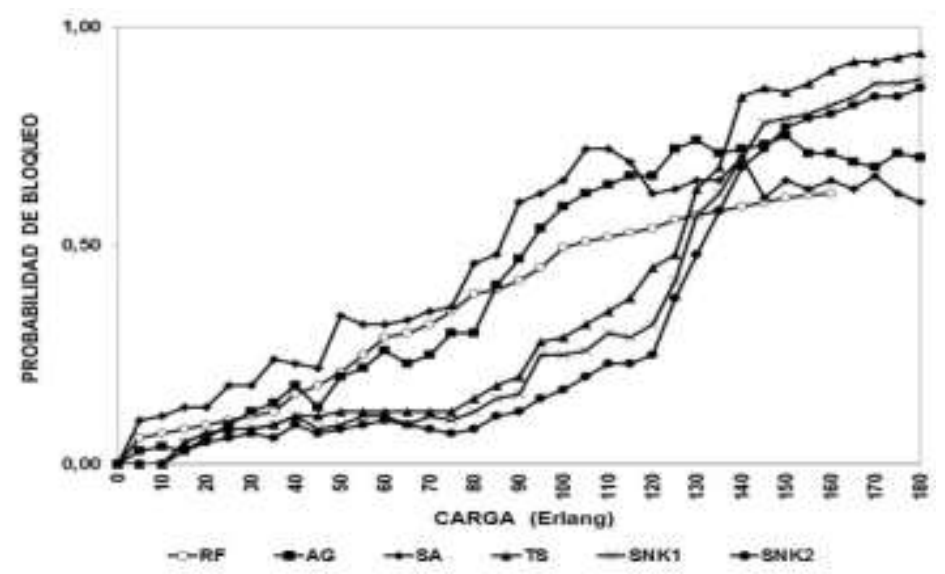

Fig. 12: Comparación de la Probabilidad de Bloqueo para cada heurística

En la Figure 13, se puede observar el aumento de la utilización de red de SNK2 a niveles bajos respecto de SNK1, esto muestra que el aprovechamiento de la estrategia de Enlace Saturado no aumenta significativamente el uso de los recursos, pero si disminuye la probabilidad de bloqueo. Para altos niveles de stress (cargas superiores a 140 Erlangs) los algoritmos AG, SA y TS logran un uso bajo de los recursos de la red en comparación con el resto de los algoritmos ensayados.

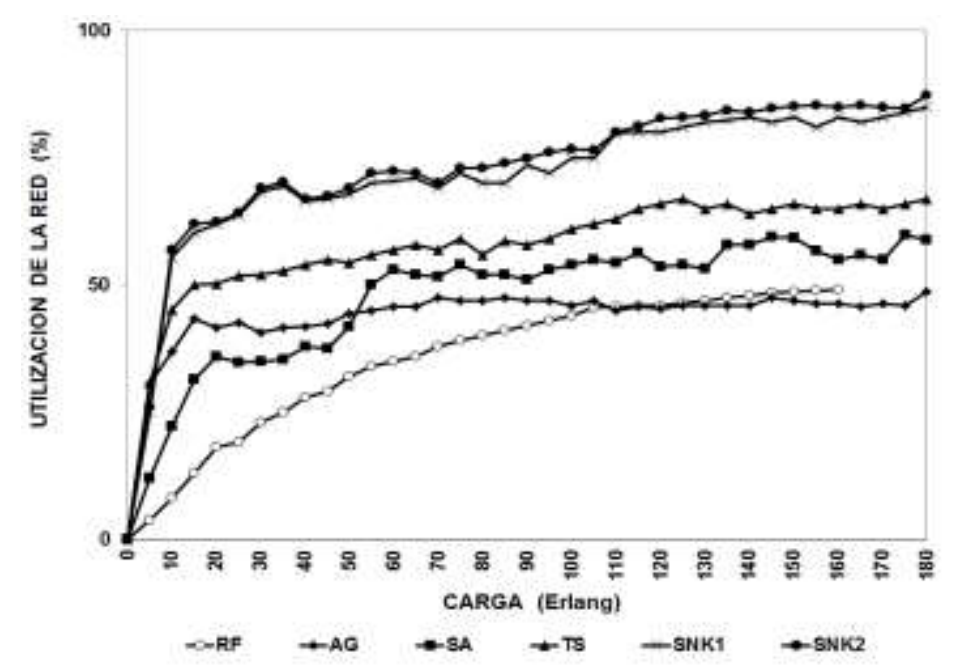

Fig. 13: Comparación de la utilización de la red para cada heurística.

\section{CONCLUSIONES}

Se logra demostrar que la incorporación de la metaheurística SNK2 logra disminuir el comportamiento de la probabilidad de bloqueo a un costo bastante bajo de la utilización de lared. La estrategia de utilizar los enlaces saturados permite el aumento del tráfico en algunas zonas y disminuya en otras. Teniendo como consecuencia una mejora de la atención a las solicitudes entrantes. Sin embargo el algoritmo continua obteniendo largos lightpath, es decir muchos saltos, lo que explica el aumento de la utilización de la red.

Cuando el tráfico se encuentra por debajo de los 140 Erlangs SNK2 obtiene su mejor comportamiento, y cuando se llega a escenarios de Stress, es decir por encima de los 140 Erlangs el algoritmo no es más robusto que le resto de heurísticas. Sin embargo, el análisis del TAH indica que es un algoritmo que tiene una bajo costo para obtener su probabilidad de bloqueo lo que determina una alta robustez.

Es necesario realizar nuevas modificaciones al algoritmo para lograr mejoras sustanciales en la probabilidad de bloqueo. El Grupo de Investigación espera realizar un nuevo ensayo monitorizando los nodos (SNK3), esperando que esto mejore los indicadores y se logre mejores comportamientos frente a cargas superiores a los 120 Erlangs. 


\section{AGRADECIMIENTOS}

Para el Programa DYCYT de la Universidad de Santiago de Chile - USACH (Project DICYT N 061572RG), por su importante apoyo al desarrollo de la investigación y al Grupo de Investigación en Nuevas Tecnologías (GINT-DTI-USACH).

\section{REFERENCIAS}

Assis, K. D. R., Ferreira dos Santos, A. y Giozza, W. F., Hybrid Algorithms for Routing and Assignment Wavelengths in Optical Networks, Latin America Transactions, 8(3), 214-220 (2010)

Bettemir, O. y Sonmez, R., Hybrid Genetic Algorithm with Simulated Annealing for Resource-Constrained Project Scheduling, Journal Of Management In Engineering, 31(5), 04014082 (2015)

Chen, M., Lin, B. y Tseng, S., Ant colony optimization for dynamic routing and wavelength assignment in WDM networks with sparse wavelength conversion, Eng. App. Artificial Intelligence, 24(2), 295-305 (2011)

Chiappone, S., Giuffre O., Grana, A., Mauro, R. y Sferlazza, A., Traffic simulation models calibration using speed-density relationship: An automated procedure based on genetic algorithm, Expert Systems With Applications, 44, 147-155 (2016)

Dong, Y., Zhao, S., Ran, H., Li, Y. y Zhu, Z., Routing and Wavelength Assignment in a Satellite Optical Network Based on Ant Colony Optimization With the Small Window Strategy,Journal Of Optical Communications And Networking, 7(10), 995-1000 (2015)

Juhari, N., Menon, P., Ehsan, A. and Shaari, S., Optical loss analysis in 13-channel SOl-based AWG for CWDM network, Journal Of Nonlinear Optical Physics \& Materials, 23(1), 1450008(9) (2015)

Pan, Y., Hui, D. y Ramamurthy, B., Cost-optimized joint resource allocation in grids/clouds with multilayer optical network architecture, Optical Communications and Networking, IEEE/OSA Journal of, 911-924 (2014)

Pandya, R., Chandra, V. y Chadha, D., Simultaneous Optimization of Power Economy and Impairment Awareness by Traffic Grooming, Mixed Regeneration, and All Optical Wavelength Conversion With an Experimental Demonstration, Journal Of Lightwave Technology, 32(24), 4166-4177 (2014)

Rodríguez, A. y Saavedra F., Optimización del Algoritmo Genético para la Solución Integral de Enrutamiento en Redes Fotónicas, Inf. Tecnol., 21(3), 125-133 (2010)

Rodriguez, A., Gutierrez, A., Rivera, L. y Ramirez, L., Ruteo y Asignación de Longitud de Onda: Comparación de Algoritmos Genéticos y Simulated Annealing, Inf. tecnol., 25(4), 13-18 (2014)

Rodriguez, A., Ramirez, L., Rivera, L. y Gutierrez, A., Routing wavelength assignement: A solution with tabu search in dynamic traffic, Ingeniare. Rev. Chil. Ing., 22(4), 495-503 (2014)

Rodríguez, A., Travieso-Torres, J. y Ramírez, L., New heuristic algorithm for dynamic traffic in WDM optical networks. Ingeniería e Investigación, 35(3), 100-106 (2015)

Rubio-Largo, A., Vega-Rodriguez, M. A., Gomez-Pulido, J.A. y Sanchez-Perez, J.M., Multiobjective Metaheuristics for Traffic Grooming in Optical Networks, Evolutionary Computation, IEEE Transactions on, 17(4), 457-473 (2013)

Rubio-Largo, A., Vega-Rodríguez, M., Gómez-Pulido, J. y Sánchez-Pérez, J., Using a Multiobjective OpenMP+MPI DE for the Static RWA Problem, Computer Aided Systems Theory, LNCS Springer-Verlag, 6927(1), 224-231 ( 2012)

Rubio-Largo, A., Vega-Rodríguez, M., Gómez-Pulido, J. y Sánchez-Pérez, J., A Multiobjective Gravitational Search Algorithm Applied to the Static Routing and Wavelength Assignment Problem, Applications of Evolutionary Computation, Lecture Notes in Computer Science, Springer-Verlag, 6625(1), 41-50 (2011)

Rubio-Largo, A., Vega-Rodríguez, M., Gómez-Pulido, J. y Sánchez-Pérez, J., Tackling the Static RWA Problem by Using a Multiobjective Artificial Bee Colony Algorithm, Advances in Computational Intelligence . Lecture Notes in Computer Science; Springer-Verlag, 6692(1), 364-371 (2011)

Xin-She, Y., Su Fong, C. y Tiew, T., Bio-Inspired Computation in Telecommunications, $1^{\circ}$ edición, 55-65, Morgan Kaufmann. Waltham, USA (2015)

Zahilah, R., Ogino, Y., Nishium, S., Nooruzzaman, M., Thuy, NTT., Koyama, O. and Katsuyama, Y., Lightpath design \& management system for IP-over-CWDM networks with ROADMs, employing parallel processing, Telecommunication Systems, 53(2), 1033-1042 (2013)

Zang, H., Jue, J., Sahasrabuddhe, L., Ramamurthy, R. y Mukherjee, B., Dynamic lightpath establishment in wavelength routed WDM networks, Communications Magazine IEEE, 39(9), 100-108, Septiembre (2001) 